\title{
QTL x environment interactions for panicle traits in switchgrass (Panicum virgatum)
}

\author{
Li Zhang ${ }^{1}$, Xiaoyu Weng ${ }^{1}$, Kathrine Behrman ${ }^{1}$, Jason Bonnette ${ }^{1}$, John Reilley ${ }^{2}$, Francis \\ Rouquette $^{3}$, Phillip Fay ${ }^{4}$, Yanqi $\mathrm{Wu}^{5}$, Felix Fritschi ${ }^{6}$, Robert Mitchell ${ }^{7}$, David Lowry ${ }^{8}$, \\ Arvid $\mathrm{Boe}^{9}$, and Thomas Juenger ${ }^{1}$ \\ ${ }^{1}$ The University of Texas at Austin \\ ${ }^{2}$ USDA National Resources Conservation Service \\ ${ }^{3}$ Texas A\&M University Agricultural Research and Extension Center at Overton \\ ${ }^{4}$ USDA-ARS Grassland Soil and Water Research Laboratory \\ ${ }^{5}$ Oklahoma State University Stillwater \\ ${ }^{6}$ University of Missouri System \\ ${ }^{7}$ USDA-ARS Wheat Sorghum and Forage Research \\ ${ }^{8}$ Michigan State University \\ ${ }^{9}$ South Dakota State University
}

November 5, 2020

\begin{abstract}
Panicle traits exhibit quantitative variation controlled by genes, the environment, and their interaction. In switchgrass, a perennial biofuel crop, identification of quantitative trait loci (QTL) and QTL x E interactions controlling panicle architecture could aid breeding efforts and cultivar development by impacting seed productivity. In this study, we evaluate the genetic architecture of panicle traits including panicle length, primary branching number, and secondary branching number in an outcrossing switchgrass population grown across ten field sites in the central United States. We evaluated pleiotropic relationships between panicle traits and flowering time, tiller production and biomass. We also identified environmental factors correlated with QTL $\mathrm{x}$ E interactions and potential candidate genes underlying panicle trait QTL in switchgrass. Overall, our multi-environment mixed QTL model detected 18 QTL for panicle traits. Twelve of the QTL exhibited consistent effects (i.e., no QTL x E), and most (4 of 6) of the effects with QTL x E exhibited condition-specific effects. Many of the QTL x E effects were associated with yearly mean temperature and photoperiod. Panicle QTL co-localized with previously identified flowering time QTL and candidate genes associated with flowering, supporting a pleiotropic model of panicle development based on shared developmental genetics and responses to environmental signals.
\end{abstract}

\section{Introduction}

As the bearers of grain, the grass panicle (or inflorescence) has been the target of selection for thousands of years (Doust, 2007). There is enormous diversity in panicle architecture within and among grass species (Coen \& Nugent, 1994). Panicle architectures are critical determinant of interspecies differences in plants morphology and life history, and are often measured as variation in panicle length, branching (number, length, and pattern), and flower number and size borne on each branch type. Simple panicles may have only primary branches, while complex panicles can possess many secondary and tertiary branches (Bommert \& Whipple, 2018; Glemin \& Bataillon, 2009). In wild grasses, branching pattern plays an important role in wind pollination and affects the number and size of seeds, which ultimately influences seed yield and plant fitness (Friedman \& Harder, 2004). In domesticated species, there is a direct association between 
panicle architecture and seed productivity (Brown et al., 2006; Crowell et al., 2016; Wang \& Li, 2005). Analysis of the phylogenetic distribution of panicle variation in the grasses suggests that different panicle architecture have arisen independently many times, and homoplasy across the grass phylogeny has obscured the mechanisms of panicle diversity (Doust \& Kellogg, 2002; Kellogg, 2000). These traits likely evolve in response to natural selection mediated by pollinator (Friedman \& Harder, 2004) and environmental variation such as light (Vogler et al., 1999), drought (Mal \& Doust, 2005), nutrient availability (Dorken \& Barrett, 2004), soil water availability (Caruso, 2006), and intraspecific competition (Wolfe \& Mazer, 2005). Given the importance of inflorescence architecture to the fitness and productivity of both wild and domesticated species, it is of great interest to understand genetic variation in panicle architecture.

Organisms often respond to changing resource availability and environmental signals through phenotypically plastic changes (Sultan, 2000). Panicle traits often display plasticity in response to different environmental cues, as panicle development involves complex regulatory mechanisms and these mechanisms interact with environmental signals (Adriani et al., 2016; Bai et al., 2016; Tu et al., 2019). For example, Adriani et al. (2016) found that secondary branch number was the most variable (or plastic) trait of panicle architecture in response to light resources in rice. Secondary branching was also found to be influenced by water deficit more than primary branching in a rice recombinant inbred family (Liu et al., 2010). Abiotic components such as heat, drought, and light affect panicle development and ultimately panicle architecture (Adriani et al., 2016; Wu et al., 2017; Wu et al., 2016; Mitchell et al, 1997). In addition to environmental effects on variation in panicle traits, standing genetic variation within species in panicle traits is also common (Jamal et al., 2009; Brown et al., 2006; Hong \& Yan, 2004; Ungerer et al., 2002).

Genetic variation in phenotypic plasticity in response to the environment is better known as genotype-byenvironment interactions (G x E) (Des Marias et al., 2013). Quantitative studies of G x E in many crops (e.g., maize, rice) have identified important quantitative trait loci (QTL) impacting many panicle traits (Kovi et al., 2011; Leng et al., 2017; Zhao et al., 2017). For example, Leng et al. (2017) identified 17 QTL for five panicle related traits in a double haploid population in rice. Among these QTL, six QTL showed QTL-byenvironment interactions, indicating that panicle related traits are susceptible to environmental influence. Zhao et al. (2017) found that 11 out of 19 QTL were involved in QTL-by-environment interactions for tassel primary branching number in maize under different watering environments. G x E is common in QTL studies and identifying $\mathrm{G} \times \mathrm{E}$ and the pattern of interactions is of great interest to understand the genetic architecture underlying phenotypic traits.

Pleiotropy is the phenomenon of a single gene affecting multiple distinct traits (Williams, 1957) and is an important driver of trait integration and modularity (Armbruster et al., 2014; Klingenberg, 2008). Pleiotropy contributes to the genetic correlation among traits and therefore has broad implications in genetics, development and adaptive evolution (Armbruster et al., 2014; Auge et al. 2019; Pigliucci and Preston 2004;). In plants, the formation of all aboveground organs, such as leaves, tiller, internodes, and inflorescences is mainly dictated by the activity and determinacy of the shoot apical and axillary meristems. During the vegetative-to-reproductive transition, many developmentally related traits originate from the same meristem and complex environmental signals may affect the different type of meristems by similar regulatory networks (Wang \& Li, 2008; Xue et al., 2020). Therefore, the loci for vegetative and reproductive development-related traits frequently show pleiotropy. For example, most flowering time pathway genes show pleiotropic effects on tiller number and yield potential in crops (Auge et al. , 2019). Genes involved in hormone pathways frequently affect both vegetative growth and reproductive development (Lee et al., 2019), in part through their developmental impacts on meristems (Azizi et al., 2015). QTL mapping is one of the many approaches that have been used to estimate genome-wide pleiotropy, and fits squarely in the context of developmental pleiotropy (Paaby \& Rockman, 2013). Pleiotropic effects identified through overlapping QTL locations have been observed in panicle development in sorghum and rice (Brown et al., 2006; Endo-Higashi \& Izawa, 2011; Komatsu et al., 2001; Miura et al., 2010; Yu et al., 2017), but these patterns have not been investigated in native perennial grasses.

Switchgrass (Panicum virgatum L.) has been championed as a potential biofuel crop since it was selected by 
the US Department of Energy (US DOE) as a model grass species for bioenergy in the early 1990s (Hohenstein \& Wright, 1994; McLaughlin, 1993). Its potential for high biomass production on marginal land, adaptation to a wide range of environments, and ecosystem service such as carbon sequestration, water flow management and erosion control, makes switchgrass an excellent candidate for filling bioenergy needs (Mitchell, Vogel, \& Uden, 2012; Robertson et al., 2017). Switchgrass is a warm-season C4 perennial grass native to the North America, with a range that extends from the eastern seaboard west to the Rocky Mountains and from southern Canada south to the Texas Coastal Plain and Northern Mexico (Casler, 2007; Hopkins, 1995). Two major distinctive populations have been classified in the past based on morphology and habit preference, northern upland and southern lowland ecotypes (Porter Jr, 1966). A very recent study based on a resequenced switchgrass diversity panel was able to define a third coastal ecotype, which is broadly sympatric with the lowland ecotype but possesses upland leaf characters and lowland plant morphotype (Lovell et al., 2020, in review).

Information on panicle morphology is limited in switchgrass, although panicle length differences have been reported between switchgrass ecotypes and cultivars (Porter Jr, 1966; Price, 2014; Van Esbroeck, 2003). We hypothesize that panicle evolution in switchgrass may be related to selection on aspects of mating system and degree of investment in vegetative versus sexual reproduction, especially in the context of seedling establishment in differing habitats. For example, lowland switchgrass has a restricted bunch grass growth form and occurs primarily in patchy distributions along riparian areas. In contrast, upland switchgrass has a rhizomatous spreading growth form that occurs in many prairie habitats. Pattern of pollen dispersal across patches, or aspects of seed establishment (e.g. seed size/number tradeoffs or disturbance regimes) likely differ in these habitats and may have driven divergence in panicle form. Panicle morphology and its relationship to seed quality may be important targets of selection and breeding, as consistent seed production will be critical to meet the demands for large-scale biofuel production (Das \& Taliaferro, 2009; Vogel, 2000).

In this study, we evaluated the genetic architecture of panicle traits across ten field sites in the central US to investigate the importance and nature of $\mathrm{G} \times \mathrm{E}$ for panicle traits. To accomplish this goal, we planted clonal divisions of progeny from a four-way outbred mapping population derived from upland and lowland germplasm, along with the four grandparents and F1 hybrids, at ten field sites spanning a large latitudinal gradient. Three panicle traits including panicle length (PL), primary branching number (PBN) per panicle, and secondary branching number (SBN) on the panicle, were assessed at each site to investigate: (1) the genetic architecture underlying these three traits, (2) the sensitivity of QTL and their effects across different environments (i.e., QTL x E), (3) the extent of pleiotropy between panicle and other traits, and (4) the environmental factors contributing to QTL x E interactions. Finally, we identified candidate genes potentially involved in regulating panicle architecture in switchgrass.

\section{Materials and Methods}

\section{Field experiment and phenotyping}

The details of the creation of this population were described in Milano et al. (2016). Briefly, the grandparents of the mapping population were derived from highly divergent southern lowland and northern upland ecotypes. The population was developed by initial crosses between AP13 (A) x DAC6 (B) and WBC3 (C) x VS16 (D). AP13 and WBC3 are genotypes clonally derived from an individual selected from the lowland cultivar 'Alamo' (southern Texas accession) and an individual from naturally occurring population 'West Bee Cave' (central Texas accession), respectively. DAC6 and VS16 are genotypes clonally derived from individuals selected from the upland cultivars 'Dacotah' and 'Summer' (both northern upland accessions), respectively. The $\mathrm{F}_{1}$ hybrids of each of those crosses were then intercrossed reciprocally to produce the four-way outbred mapping population.

The grandparents, $F_{1}$ hybrid parents, and the $F_{2}$ progeny were propagated by dividing plants manually to produce 10 clones, each of which was maintained in a 3.8-L pot at the Brackenridge Field Laboratory, Austin, TX in 2013-2015. One replicate of each of the mapping progeny genotypes, along with multiple replicates of grandparents and $F_{1}$ parents, were transplanted from May to July of 2015 at 10 field sites. In three of 
the 10 sites, 370 extra genotypes were planted. The 10 field sites cover 17 degrees of latitude from South Texas to South Dakota (Figure 1A). Detailed information of the 10 field sites, including latitude, longitude and soil type is provided in Table 1. The annual mean temperature at the 10 sites in 2016 ranged from $10.4{ }^{\circ} \mathrm{C}$ in the north to $20.7^{\circ} \mathrm{C}$ in the south, and the total rainfall varied from $574 \mathrm{~mm}$ to $1440 \mathrm{~mm}$ (Figure $1 \mathrm{~B}$, data are from local weather station or from NOAA if local weather data are not available; the weather station or NOAA link is included in Table 1). To control weeds, each field site was covered with one layer of weed barrier cloth (Dewitt, Sikeston, MO). Holes were cut into the weed cloth in a honeycomb fashion. Plants were randomized into the holes, with each plant having four nearest neighbors each located $1.56 \mathrm{~m}$ away from each other. A row of border plants was planted at every edge position of the field to minimize edge effects. The border plants were derived from rhizome plugs obtained from an approximately 10-year-old stand of Alamo switchgrass. Plants were well watered in the field during the summer of 2015 to facilitate establishment and all phenotypes were collected in 2016.

Three panicles were cut from each plant at full maturity. Panicle length (PL in mm), primary branching number $(\mathrm{PBN})$, and secondary branching number (SBN) were assessed at the end of the growing season. A diagram depicting these phenotypes is presented in Figure 2, with representative images of panicles from the four grandparents. PL was measured on the primary panicle from the base of the first primary branch to the top of the panicle. PBN was counted as the total number of branches along the primary rachis. Due to the numerous secondary branches in switchgrass, SBN in our study referred to the total number of secondary branches on the lowest primary branch of the panicle (Figure 2). In total, over 10,000 separate panicle morphology measurements were collected in our study. The phenotypic data (i.e., average values) for each genotype at each field site are provided in Supplemental Table S1.

\section{Genotyping and multi-environment QTL modelling}

Illumina fragment paired end libraries, representing each of the four grandparents (A: AP13, B: DAC; C: WBC; D: VS16) were sequenced and genotyped in the context of the P.virgatum reference genome v5 as detailed in (Bragg et al., 2020). The genetic map spans 750 recombinant 4-way progeny genotyped at 4700 markers. Details on the genetic map construction, map polishing and fine-scale reordering can be accessed on https://datadryad.org/stash/dataset/doi:10.5061/dryad.ghx3ffbjv (Lovell et al., 2020). For computational efficiency in $\mathrm{G} \times \mathrm{E}$ analysis, the genetic map was reduced into 738 markers, with an average intermarker distance of to $2 \mathrm{cM}$.

Narrow-sense heritability $\left(h^{2}\right)$ for each trait at each environment and the genetic correlation between traits among sites and across sites were estimated using the additive kinship matrix based on marker genotypic information using the 'sommer' package (Covarrubias-Pazaran, 2016) in R (2018). Briefly, we used a multivariate mixed model (mmer) that takes the kinship matrix to estimate the variance components for each trait under each environment, and calculates $h^{2}$ as the proportion of additive genetic variance to the total variance.

Details of the mapping scheme and application in the outbred four-way population are described in Malosetti et al. (2014) and Lowry et al. (2019). In brief, 'single trait under multiple environments' QTL mapping for each panicle trait in the cross-pollinated (CP) family was implemented in Genstat (2019). The QTL approach with CP family resulted in four possible QTL alleles designated $A$ and $B$ corresponding to marker alleles of the first pair of grandparents (AP13 x DAC) and QTL alleles $C$ and $D$ corresponding to marker alleles of the second pair of grandparents (WBC x VS16). A multienvironment mixed model was fit for each trait as shown in Eq. 1:

trait $=u+E+\sum \mathrm{QTL}+\sum(\mathrm{QTL} \times \mathrm{E})+e \mathrm{Eq} .(1)$,

where $\mu$ is the population mean; $E$ represents the environment effect; $\sum \mathrm{QTL}=\sum\left(a^{a 1}+a^{a 2}+a^{d}\right)$, denoting the total effect from the additive effect from the first grandparent (i.e., the difference between $A$ and $B$ alleles, $a^{a 1}$ ), the second grandparent (i.e., the difference between $C$ and $D$ alleles, $a^{a 2}$ ), and the dominance effect (i.e., the intralocus interaction, $\left.a^{d}\right) ; \sum($ QTL $\times$ E) represents the QTL $\times$ environment interactions; and $e$ represents the error term that was modeled by an unstructured variance-covariance matrix. The 
unstructured model was used to specify the data structure in the genome-wide QTL scan of simple interval mapping (SIM) and composite interval mapping (CIM). A backward selection procedure was used to retain significant fixed terms $(p<0.05)$ after three consecutive runs of CIM to confirm stability of QTL. The QTL with highest LOD peaks were considered as the most significant QTL, and the flanking markers associated with 1.5 LOD drop around the most significant QTL were considered as confidence interval for the QTL peaks.

The quality of the predictions from the full G x E model (Eq. 1) was evaluated with an independent dataset. In three of the 10 field sites (CLMB, KBSM, PKLE), 370 extra genotypes were planted and panicle morphology data were collected for these genotypes in 2016. The multi-environment QTL x E model was evaluated by 1) extracting the final full model from Genstat based on the core set of progeny, 2) reconstructing the model in $\mathrm{R}, 3)$ predicting the panicle traits for these additional genotypes at these 3 field sites, and 4) comparing the model predictions with field observations. Percentage of bias (bias\%) and the prediction accuracy $(r$, or correlation coefficient) between the model predictions and field observations were used as statistical measures for model performance.

Our Genstat modeling of QTL x E considered sites as fixed effect. To further explore the possible environmental drivers of the QTL x E, we explored a variety of regression models using best subset generalized linear models ('bestglm' package, McLeod et al., 2020) in R. Here, the QTL effects (i.e., the difference of $\mathrm{A}$ versus $\mathrm{B}$ or $\mathrm{C}$ versus $\mathrm{D}$ alleles) were predicted with the yearly mean temperature (Tmean), the yearly total rainfall (Rainfall), the day length (DL), and the yearly average solar radiation (SRAD) as predictors as shown in Equation 2.

effect $=\mu+$ Tmean + Rainfall + DL + SRAD + e Eq. (2)

These environmental variables were selected because temperature, water availability and light have been identified as interacting with panicle development, as mentioned in the Introduction. Interaction terms between variables were not included in our models because they were highly correlated with the individual variable. We used the bestglm function and performed all-subset linear regression based on Bayesian information criteria (BIC) to find the best subset of variables to fit the model. Environmental variables retained from the model were considered as candidate environmental factors that interacted with specific QTL, resulting in different effects at different sites.

Additionally, to investigate potential pleiotropic relationships between panicle traits and other traits, we ran the multi-environment QTL model for flowering (FL50), tiller count (TC), and biomass (BIO) in switchgrass and examined if the QTL identified for panicle traits share the same QTL with these traits. FL50, TC and BIO data were collected in the same year as panicle data and previously reported in Lowry et al. (2019). FL50 is the day of the year when $50 \%$ of the tillers on a plant have panicles that have begun flowering. TC and BIO are the total tiller count and dry biomass at the end of the growing season, respectively. Traits with overlapping QTL confidence interval were considered as potentially pleiotropic.

\section{Candidate genes and GO enrichment analysis}

The genes located in the confidence intervals of the discovered QTL were considered candidate genes. All candidate genes were compared with the rice (v7) and Arabidopsis annotation databases (TAIR 10), and annotated with Gene Ontology (GO). The annotation file for switchgrass was accessed on JGI (Joint Genome Institute) Phytozome 13 website: https://njp-spin.jgi.doe.gov/. The GO enrichment analysis was tested using fisher's exact test for each GO term using R package 'topGO' (Alexa and Rahnenuhrer, 2019). The GOs with adjusted $p$ values of less than 0.05 were considered as significant.

\section{Results}

\section{Phenotypic variation and heritability}

There was a general trend of increasing trait values in the $\mathrm{F}_{2}$ with latitude, exhibiting latitudinal plasticity of the measured panicle traits (Figure 3). The violin plot for each trait at each site displayed approximately 
normal distribution and transgressive behavior in the $\mathrm{F}_{2}$ generation. Lowland genotypes, AP13 and WBC, always had larger values of panicle length (PL in $\mathrm{mm}$ ), primary branching number (PBN), and secondary branching number (SBN) than upland genotypes, DAC and VS16 (Figure 3).

The heritability $\left(h^{2}\right)$ for PL, PBN and SBN varied by site (Table 2). The $h^{2}$ for PL ranged from 0.20 to 0.71 , with an average of 0.46 and values greater than 0.50 at four northern sites. The $h^{2}$ for PBN ranged between 0.45 and 0.66 for 9 out of the 10 sites, with Stillwater, OK (STIL) having low heritability $\left(h^{2}=0.20\right)$. The $h^{2}$ for SBN ranged from 0.02 to 0.62 , where Stillwater, OK (STIL) had $h^{2}$ close to zero $\left(h^{2}=0.02\right)$, Columbia, MO (CLMB) had low heritability $\left(h^{2}=0.15\right)$, and four sites had heritability at approximately 0.50 . These changes in heritability by environment indicate $\mathrm{G} \times \mathrm{E}$, which primarily is the result of changes in variances across the common garden environments.

The phenotypic and genetic correlations between traits were generally positive but varied by site, ranging from 0.21 to 0.63 for phenotypic correlation and from 0.35 to 0.88 for genetic correlation (Table 3 ). The phenotypic correlation between traits across all sites ranged from 0.50 to 0.61 . The genetic correlation between PL and PBN across all sites was close to zero (0.03), while the genetic correlation between PBN and SBN was high (0.73) (Table 3). Together, these results suggest independence in panicle length and branching characteristics.

\section{Multi-environment mixed QTL model}

A total of 18 QTL were identified for panicle morphology traits with the multi-environment mixed model analyses (Figure 4, Table 4). Seven QTL were identified for PL, distributed across seven different chromosomes. Among these, five QTL (2K@77.89, 4K@26.26, 5K@76.02, 5N@36.27 and 9N@38.02) had consistent effects across field sites (Figure 5a). In contrast, two QTL (3N@62.06 and 6N@54.19) show interaction with the environment (QTL x E). The additive effects for QTL 3N@62.06 changed in magnitude across geographic regions. Further, QTL 6N@54.19 had the largest effects at the most northern and southern site and smaller effect at mid-latitude sites. QTL 6N@54.19 (A x B cross) also had a trade-off pattern, with the allelic effects changing sign from southern to northern sites.

Seven QTL were identified for PBN that are also distributed on 7 chromosomes. Four QTL (2K@74.02, 2N@66.12, 5N@84.04 and 9N@26.03) had consistent effects across locations, while three QTL (3K@38, 5K@14.06, and 7N@54.06) had QTL x E interactions, including both changes of magnitude (3K@38 and $7 \mathrm{~N} @ 54.06)$ and direction (5K@14.06) from the allelic effect across geographic regions (Figure 5b). Four QTL were identified for SBN. Three QTL (2N@72.03, 5K@95.5 and 9N@36.02) had consistent effects across locations, while there was a magnitude changing interactions for QTL 9K@51.96 (Figure 5c). We also observed that two QTL for PBN (2K@74.02 and 9N@26.03) co-localized with PL QTL on chromosome 2K and 9N, based on overlapping confidence intervals (Figure 4). QTL 9N@38.02 for SBN co-localized with the QTL of PL and PBN on chromosome 9N (Figure 4).

\section{Pleiotropic effect}

We hypothesized that loci impacting panicle traits might show strong pleiotropic relationships with other important characteristics of switchgrass growth and development. To test this idea we examined whether the QTL identified for panicle traits shared the same QTL with flowering (FL50), tiller count (TC), and biomass (BIO) in switchgrass. Our results (Figure 4 and Table 4) showed that two QTL (2K@77.89, 3N@62.06) for PL overlapped with QTL for BIO, one QTL (4K@26.26) overlaps with FL50, and one QTL on 9N@38.02 clusters with FL50, TC, and BIO. Out of the seven QTL for PBN, six QTL shared the same QTL with either FL50, TC or BIO, with five QTL (2N@66.12, 3K@38, 5N@84.04, 7N@54.06, 9N@26.03) exhibiting pleiotropy with FL50, two QTL (3K@38, 5N@84.04) with TC and one (2K@74.02) with BIO. One QTL of SBN (9N@38.02) clustered with QTL of TC and BIO. Among these pleiotropic QTL, some showed similar patterns of QTL $\mathrm{x}$ E, providing further support for a pleiotropy hypothesis. For example, the QTL 3N@62.06 for PL exhibited condition-specific effects (Figure 5) and the overlapping QTL for BIO showed a similar pattern (data not shown). The QTL 9N@38.02 for PL displayed the same pattern (i.e., no QTL x E, figure 5) as the overlapping QTL for FL50 and BIO, but not for TC, suggesting there may be different loci controlling PL and TC. 


\section{QTL model evaluation}

The evaluation of the QTL and QTL x E model (Eq. 1) with the data collected on extra genotypes at the three sites showed low to moderate prediction accuracy (Figure 6). Panicle length had relatively low prediction accuracy $(r=0.34)$ but also low percentage of bias (\%bias $=0.3 \%$ ). Primary branching number had similar results as panicle length $(r=0.36, \%$ bias $=0.3 \%)$. Secondary branching number had a moderate prediction accuracy $(r=0.62)$ but with slightly larger percentage of bias (\%bias $=0.6 \%)$.

\section{Environmental factor exploration}

To evaluate possible environmental drivers of $\mathrm{G}$ x E, we used a regression-based approach. Here, we evaluated yearly mean temperature, annual total rainfall, day length (photoperiod), and yearly average solar radiation as possible drivers of QTL x E for each of the six QTL with site interactions. Overall, the main drivers of the QTL x E identified in this study are yearly mean temperature and photoperiod, with impacts on eight QTL (Table 5). Rainfall was also identified as a driver of the QTL x E for the QTL 6N@54.19 of LEN and $5 \mathrm{~K} @ 14.06$ of PBN, and solar radiation as a driver of the QTL $\mathrm{x}$ E for the QTL 9K@51.96 of SBN (Table 5). We failed to identify candidate environmental factors for some of the QTL $\mathrm{x}$ E. This is likely related to the fact that not all the environmental factors (such as soil) were accounted for in our analyses.

\section{Candidate gene identification and GO enrichment analysis}

Panicle traits have been well-studied in crop plants and model systems and many candidate genes have been reported (Miura et al., 2010; Doust, 2007; McSteen, 2006; Vollbrecht et al., 2005). For the 18 panicle architecture QTL identified in our study, the confidence intervals ranged from 2 to $32 \mathrm{cM}$ and from 0.9 to 35 $\mathrm{Mb}$. We linked the QTL discovered here with known candidate genes that have been reported in previous studies (Supplemental Table S2).

Among these candidate genes, key transcription factors and hormone related genes associated with panicle development were identified in the intervals of most QTL exhibiting environmental interactions. For example, key regulators involved in GA metabolism (Pavir.3KG352627 as the homolog of GA2ox3 in 3K@38; Pavir.5KG065800 as the homolog of GA3ox2 in 5K@14.06) and CK signaling pathways (Pavir.7NG435700 as the homolog of ARR6 in 7N@54.06; Pavir.9KG213000 as the homolog of ARR1 in 9K@51.96) were found in four branching QTL x E intervals. A number of key flowering genes were also found in our panicle QTL intervals. These genes are known to be involved in the photoperiodic flowering pathway and control panicle morphology in other grasses (Shrestha et al., 2014; Tsuji et al., 2010). However, these candidate genes were not co-localized with flowering QTL that we identified, indicating the potentially complex functions of flowering time genes in the transition from vegetative to reproductive phases.

GO enrichment analysis identified 380 significant GO terms for genes within the QTL intervals for panicle traits. 'Response to Auxin' was one of the significantly enriched GO terms $(p=0.0012)$. This is an exciting result as the auxin signaling pathway has been previously shown to be important in panicle meristem development and affects primary branching number in rice and other grasses (He et al., 2018; Zhang \& Yuan, 2014). Several other significant terms that are relevant to our study traits include 'response to oxidative stress' and 'H4/H2A histone acetyltransferase complex' (Boycheva, Vassileva, \& Iantcheva, 2014; Deng et al., 2007; Peng et al., 2018; Wu et al., 2017; Yano et al., 2019). These results point to potentially interesting candidate genes and hormone-related pathways that are likely important in panicle development.

\section{Discussion}

There has been considerable interest in the molecular mechanisms of $\mathrm{G}$ x E across a diversity of phenotypes, species, and environments. G x E is common and is often driven by differential sensitivity of alleles and may play an important role in adaptive plasticity and local adaptation (Des Marais et al., 2013). With its large scale, our study evaluated the genetic basis and examined the QTL x E of panicle morphological traits in switchgrass grown at 10 field sites in the central United States (Figure 1). Overall, we detected moderate heritability (except for the field site Stillwater, OK) for panicle traits (Table 2) and positive phenotypic and genetic correlations between traits at each site and across sites (Table 3). These data suggest considerable 
standing genetic variation in inflorescence characteristics available for natural or artificial selection to act upon. We identified several QTL with significant QTL x E effects and the potential environmental factors underlying the QTL x E, indicating that panicle traits in switchgrass result from the combination of QTL and environment. We also detected pleiotropic effects between panicle traits and flowering time as well as tiller count and biomass, suggesting a possible shared genetic basis between different traits.

Our study identified genomic regions (QTL) that contribute to panicle trait variation across a broad latitudinal gradient. These QTL exhibited constant effects (i.e., no QTL x E), antagonistic pleiotropy, or condition-specific effects across the studied environmental gradients. QTLs with condition-specific effects are relatively easy to incorporate into breeding programs because the selected favorable alleles will confer an advantage in some environments, without a negative effect in other environments (El-Soda et al., 2014). Antagonistic pleiotropy is a genetic trade-off at an individual locus or QTL, that results in opposite effects (i.e., sign change) on a trait in different environments (Wadgymar et al., 2017). QTL with antagonistic pleiotropy can result in tradeoffs and challenges in breeding if the preferred allele depends strongly on the environment (El-Soda et al., 2014; Lowry et al., 2019). Studying the molecular genetic basis of specific QTL should greatly contribute to the mechanistic understanding of such QTL x E. In our study, most of the QTL are conditionally neutral. This is consistent with a recent meta-analyses which found that asymmetry of QTL effect is more often caused by conditional neutrality than it is by trades-offs (Wadgymar et al., 2017). Overall, our results show that panicle traits are controlled by a combination of QTL and the environment and, in a number of cases, their complex interaction with the environment.

Inflorescence architecture is influenced by the vegetative-to-reproductive phase transition, which also largely determines patterns of vegetative growth and resource allocation. In our study, 11 of 18 inflorescence QTLs co-localized with flowering time or vegetative growth genomic intervals, which supports the hypothesis that pleiotropy impacts the phenotypic integration of these vegetative and reproductive structures. An exciting opportunity lies in the search for the candidate genes that may underlie this integration. Fortunately, extensive genetic mapping efforts in crops and model systems have identified a number of candidate genes and a basic understanding of their role in the development of the inflorescence. For example, a locus on chromosome $9 \mathrm{~N}$ (at $38.02 \mathrm{cM}$ ) was associated with the whole process of vegetative-to-reproductive transition (PL, PBN, SBN, FL50, TC and BIO). This QTL cluster is in the vicinity of homologs of OsCOL 10 and $O s T B 1$ , which are known as the key regulators in flowering and branch development (Tan et al., 2016; Takeda et al., 2003). Specifically, $\mathrm{OsCOL}_{10}$ functions as a flowering time repressor downstream of Ghd7 and the OsTB1 gene negatively regulates lateral branching in rice. Moreover, the locus on chromosome $3 \mathrm{~K}$ (at $38 \mathrm{cM})$ was clustered with QTLs for PBN, FL50 and TC. Significantly, this QTL clustering region had large effects for PBN, suggesting a major QTL that coordinates vegetative and reproductive processes. We identified a homolog of GA2ox3 in this region, which is considered as a key factor in gibberellin catabolism and plays a central role in plant development (Sakamoto et al., 2004). These results imply that there may be a shared genetic basis between vegetative and reproductive divergence within switchgrass populations.

The low to moderate prediction accuracy (0.34-0.62) of the multienvironment mixed model (Eq. 1) is likely due to two factors. Our model only accounts for significant QTL, while there are likely many smaller QTL, that were below the threshold for detection, which contribute to variation in these traits. Unfortunately, our power to detect these small effects is likely low due to our modest sample sizes (380 progeny). Additionally, the QTL model does not consider epistatic effects or dominance effects between QTL. Epistasis is known to be an important factor that affects genetic variation and phenotypic expression in populations, especially for developmentally regulated traits like inflorescence architecture. Epistatic effects on panicle related traits have been identified in several studies (Leng et al. , 2017; Ye et al ., 2009). Further inclusion of epistasis into the multi-environment QTL model may help improve model prediction. However, our approach provides a way of predicting the performance of new genotypes under environments similar to the tested environments, and can potentially help with suitable genotype selection for traits of interest under a specific environment.

Temperature and photoperiod were the most significant predictors of QTL x E interactions. This is consistent with the pattern of additive effects of most of the QTL (Table 3), where QTL displayed conditional 
neutrality with effects either in the northern or the southern sites (Figure 5). Previous study also showed that temperature-based growing degree days and photoperiod affected switchgrass morphology (Mitchell et al., 1997). Solar radiation was also a significant driver of QTL x E for secondary branching number (SBN) QTL. This is consistent with a rice study in which SBN was more plastic in response to different light resources (Adriani et al., 2016). No environmental factors were detected for some of the QTL x E interactions, possibly because the appropriate environmental factors (i.e., growing degree days, soil moisture etc.) were not explored or were obscured by complex interactions between environmental factors. The relative low heritability for panicle traits at the field site Stillwater, OK (STIL, Table 2) also suggested that there may be other environmental factor affecting panicle traits but not being accounted for, such as effective soil moisture. As noted in Table 1, Stillwater, OK, Overton, TX (OVTN), and Manhattan, KS (MNHT) all have sandy loam soil which may impact water status and subsequently plant growth and organ expansion. In our study year, OVTN received ample rain $(\sim 1400 \mathrm{~mm})$, MNHT had slightly cooler temperature and received approximately $1000 \mathrm{~mm}$ rain, while STIL only received around $700 \mathrm{~mm}$ rain (Figure 1 ). This study could be expanded in the future to include more field sites, multiple years, and more environmental data collection such as soil composition and nutrient availability to better capture the environmental drivers underlying the QTL x E interactions and trait plasticity across large geographic regions and across multiple years.

In summary, our results suggest that variation of panicle traits in switchgrass is due to a combination of QTL and the environment, with QTL displaying different effects across geographic regions. Future work focusing on identifying the driver of QTL by environment interactions and understanding the mechanisms underlying them will facilitate the selection of suitable genotypes for specific environments in switchgrass breeding programs.

\section{Data Availability and Supplemental Files}

The phenotyping data (panicle length, PL; primary branching number, PBN; and secondary branching number, SBN) for genotypes at each of the 10 field sites (Table S1), and the candidate gene lists (Table S2) are included in the supplemental excel files.

\section{Acknowledgements}

We thank the numerous field technicians, students, and postdocs who worked in the field helping out with data collection. Special thanks go to Alice MacQueen and other Juenger Lab members for helping improve the manuscript with their comments. This research was supported and funded by the National Science Foundation Plant Genome Research Program (IOS-1444533) and by the US Department of Energy, Office of Science, Office of Biological and Environmental Research Award DESC0014156 to T.E.J. This material is based upon work supported in part by the Great Lakes Bioenergy Research Center, U.S. Department of Energy, Office of Science, Office of Biological and Environmental Research under Award Numbers DESC0018409 and DE-FC02-07ER64494. Support for this research was provided by the National Science Foundation Long-term Ecological Research Program (DEB 1832042) at the Kellogg Biological Station and by Michigan State University AgBioResearch. We thank the Joint Genome Institute and collaborators for prepublication access to thePanicum virgatum v5 AP13 genome reference.

\section{References}

Adriani, D. E., Dingkuhn, M., Dardou, A., Adam, H., Luquet, D., \& Lafarge, T. (2016). Rice panicle plasticity in Near Isogenic Lines carrying a QTL for larger panicle is genotype and environment dependent.Rice, 9 (1), 28. doi:10.1186/s12284-016-0101-x

Azizi, P., Rafii, M. Y., Maziah, M., Abdullah, S.N.A., Hanafi, M. M., Latif, M. A., Rshid, A. A., \& Sahebi, M. (2015). Understanding the shoot apical meristem regulations: A study of the phytohormones, auxin and cytokinin, in rice. Mechanisms of Development, 135, 1-15.

Auge, G. A., Penfield, S., and Donohue, K. (2019). Pleiotropy in developmental regulation by floweringpathway genes: is it an evolutionary constraint. New Phytologist, 224: 55-70. 
Bai, X., Zhao, H., Huang, Y., Xie, W., Han, Z., Zhang, B., . . . Xing, Y. (2016). Genome-Wide Association Analysis Reveals Different Genetic Control in Panicle Architecture Between Indica and Japonica Rice.The Plant Genome, 9 (2). doi:10.3835/plantgenome2015.11.0115

Bommert, P., \& Whipple, C. (2018). Grass inflorescence architecture and meristem determinacy. Seminars in Cell \& Developmental Biology, 79 , 37-47. doi:https://doi.org/10.1016/j.semcdb.2017.10.004

Boycheva, I., Vassileva, V., \& Iantcheva, A. (2014). Histone acetyltransferases in plant development and plasticity. Current genomics, 15 (1), 28-37. doi:10.2174/138920291501140306112742

Bragg, J., Tomasi, P., Zhang, L. et al. (2020). Environmentally responsive QTL controlling surface wax load in switchgrass. Theor Appl Genet, 133, 3119-3137.

Brown, P. J., Klein, P. E., Bortiri, E., Acharya, C. B., Rooney, W. L., \& Kresovich, S. (2006). Inheritance of inflorescence architecture in sorghum. Theoretical and Applied Genetics, 113 (5), 931-942. doi:10.1007/s00122-006-0352-9

Caruso, C. M. (2006). Plasticity of inflorescence traits inLobelia Diphilitica (Lobeliaceae) in response to soil water availability. American Journal of Botany, 93(4): 531-538.

Casler, M. D. (2007). Genetic Diversity, Plant Adaptation Regions, and Gene Pools for Switchgrass. Crop Science, v. 47 (no. 6), pp. 2261-2260-2007 v.2247 no.2266. doi:10.2135/cropsci2006.12.0797

Coen E. S., \& Nugent J. M. (1994). The evolution of flowers and inflorescences. Development (Suppl.), 107118.

Covarrubias-Pazaran, G. (2016). Genome-Assisted Prediction of Quantitative Traits Using the R Package sommer. PLOS ONE, 11 (6), e0156744. doi:10.1371/journal.pone.0156744

Crowell, S., Korniliev, P., Falcão, A., Ismail, A., Gregorio, G., Mezey, J., \& McCouch, S. (2016). Genomewide association and high-resolution phenotyping link Oryza sativa panicle traits to numerous trait-specific QTL clusters. Nature Communications, 7 (1), 10527. doi:10.1038/ncomms10527

Das, M. K., \& Taliaferro, C. M. (2009). Genetic variability and interrelationships of seed yield and yield components in switchgrass.Euphytica, 167 (1), 95-105. doi:10.1007/s10681-008-9866-3

Deng, W., Liu, C., Pei, Y., Deng, X., Niu, L., \& Cao, X. (2007). Involvement of the Histone Acetyltransferase AtHAC1 in the Regulation of Flowering Time via Repression of \&lt;em\&gt;FLOWERING LOCUS C\&lt;/em\&gt; in Arabidopsis. Plant Physiology, 143 (4), 1660. doi:10.1104/pp.107.095521

Doust, A. \& Kellogg, E. A. (2002). Inflorescence diversification in the panicoid 'bristle grass' clade (Paniceae, Poaceae): Evidence from melecular phylogenies and developmental morphology. American Journal of Botany, 89(8): 1203-1222.

Dorken, M. E., \& S. C. H. Barrett. 2004. Phenotypic plasticity of vegetative and reproductive traits in monoecious and dioecious populations of Sagittaria latifolia (Alismataceae): a clonal aquatic plant. Journal of Ecology , 92: 32-44.

Doust, A. (2007). Architectural evolution and its implications for domestication in grasses. Ann Bot, 100 (5), 941-950. doi:10.1093/aob/mcm040

El-Soda, M., Malosetti, M., Zwaan, B. J., Koornneef, M., \& Aarts, M. G. M. (2014). Genotype × environment interaction QTL mapping in plants: lessons from Arabidopsis. Trends in Plant Science, 19 (6), 390-398. doi:https://doi.org/10.1016/j.tplants.2014.01.001

Endo-Higashi, N., \& Izawa, T. (2011). Flowering time genes Heading date 1 and Early heading date 1 together control panicle development in rice.Plant \& cell physiology, 52 (6), 1083-1094. doi:10.1093/pcp/pcr059

Friedman, J., \& Harder, L. D. (2004). Inflorescence architecture and wind pollination in six grass species. Functional Ecology, 18 (6), 851-860. doi:10.1111/j.0269-8463.2004.00921.x 
Glemin, S., \& Bataillon, T. (2009). A comparative view of the evolution of grasses under domestication. New Phytol, 183 (2), 273-290. doi:10.1111/j.1469-8137.2009.02884.x

He, Q., Yang, L., Hu, W., Zhang, J., \& Xing, Y. (2018). Overexpression of an auxin receptor OsAFB6 significantly enhanced grain yield by increasing cytokinin and decreasing auxin concentrations in rice panicle. Scientific Reports, 8 (1), 14051. doi:10.1038/s41598-018-32450-х

Hohenstein, W. G., \& Wright, L. L. (1994). Biomass energy production in the United States: an overview. Biomass and Bioenergy, 6 (3), 161-173. doi:https://doi.org/10.1016/0961-9534(94)90073-6

Hong, D., \& Yan, L. (2004). Genetic Analysis of Heterosis for Number of Spikelets per Panicle and Panicle Length of F1 Hybrids in japonica Rice Hybrids. Rice Science , 11(5-6): 255-260. Hopkins, A. A. (1995). Genotypic Variability and Genotype $\times$ Environment Interactions among Switchgrass Accessions from the Midwestern USA. Crop Science, v. 35 (no. 2), pp. 565-560-1995 v.1935 no.1992. doi:10.2135/cropsci1995.0011183X003500020047x

Kellogg, E. A. (2000). Molecular and morphological evolution in the Andropogoneae. In S. W. L. Jacobs and J. Everett [eds.],Grasses: systematics and evolution ,149-158.CSIRO,Melbourne,Australia..

Klingenberg, C. P (2008). Morphological integration and developmental modularity. Annual Review of Ecology, Evolution, and Systematics , 39 (1): 115-132.

Komatsu, M., Maekawa, M., Shimamoto, K., \& Kyozuka, J. (2001). The LAX1 and FRIZZY PANICLE 2 Genes Determine the Inflorescence Architecture of Rice by Controlling Rachis-Branch and Spikelet Development.Developmental Biology, 231 (2), 364-373. doi:https://doi.org/10.1006/dbio.2000.9988

Leng, Y., Xue, D., Huang, L., Chen, L., Ren, D., Yang, Y., . . Zeng, D. (2017). Mapping QTL with main effect, digenic epistatic and QTL $\times$ environment interactions of panicle related traits in rice (Oryza sativa). International Journal of Agriculture and Biology, 19 (6), 1608-1614.

Li, H., \& Durbin, R. (2009). Fast and accurate short read alignment with Burrows-Wheeler transform. Bioinformatics, 25 (14), 1754-1760. doi:10.1093/bioinformatics/btp324

Liu, G., Mei, H., Liu, H. et al. (2010). Sensitivities of rice grain yield and other panicle characters to late-stage drought stress revealed by phenotypic correlation and QTL analysis. Mol Breeding, 25, 603-613.

Lovell, J., Healy A., Schmutz J. and Juenger T. (2020). Switchgrass v5 4-way (AP13 x DAC, WBC x VS16) genetic map v2, Dryad, Dataset. http://doi.org/10.5061/dryad.ghx3ffbjv

Lovell, J., MacQueen, A., et al. (2020). Multiple genomic paths to climate adaptation in the biofuel crop, switchgrass. Nature (in review).

Lowry, D. B., Lovell, J. T., Zhang, L., Bonnette, J., Fay, P. A., Mitchell, R. B., . . Juenger, T. E. (2019). QTL $\times$ environment interactions underlie adaptive divergence in switchgrass across a large latitudinal gradient. Proceedings of the National Academy of Sciences, 116 (26), 12933. doi:10.1073/pnas.1821543116

Mal, T. K., \& J. Lovett-Doust. 2005. Phenotypic plasticity in vegetative and reproductive traits in an invasive weed, Lythrum salicaria (Lythraceae), in response to soil moisture. American Journal of Botany, 92: 819-825.

McLaughlin, S. (1993). New switchgrass biofuels research program for the southeast. In: Proceedings of the annual automative technology development contractors coordinating meeting, Nov. 2-5, 1992, Dearborn , $111-115$.

McSteen, P. (2006). Branching out: The ramosa pathway and the evolution of grass inflorescence morphology. The Plant Cell , 18(3): 518-522.

Milano, E. R., Lowry, D. B., \& Juenger, T. E. (2016). The Genetic Basis of Upland/Lowland Ecotype Divergence in Switchgrass (Panicum virgatum). G3 (Bethesda, Md.), 6 (11), 3561-3570. doi:10.1534/g3.116.032763 
Mitchell, R., Moore, K., Moser, L., Fritz, J., \& Redfearn, D. (1997). Predicting developmental morphology in switchgrass and big bluestem. Agronomy Journal, 89, 827-832.

Mitchell, R., Vogel, K. P., \& Uden, D. R. (2012). The feasibility of switchgrass for biofuel production. Biofuels, 3 (1), 47-59. doi:10.4155/bfs.11.153

Miura, K., Ikeda, M., Matsubara, A., Song, X. J., Ito, M., Asano, K., . . Ashikari, M. (2010). OsSPL14 promotes panicle branching and higher grain productivity in rice. Nat Genet, 42 (6), 545-549. doi:10.1038/ng.592

Paaby, A. B., \& Rockman, M. V. (2003). The many faces of pleiotropy. Trends in genetics , 29 (2), 66-73.

Peng, Y., Hou, F., Bai, Q., Xu, P., Liao, Y., Zhang, H., . . . Wu, X. (2018). Rice Calcineurin B-Like Protein-Interacting Protein Kinase 31 (OsCIPK31) Is Involved in the Development of Panicle Apical Spikelets.Frontiers in plant science, 9 , 1661-1661. doi:10.3389/fpls.2018.01661

Pigliucci, M. \& Preston, D. (eds). 2004. Phenotype integration: studying the ecology and evolution of complex phenotypes. Oxford Press, New York

Porter Jr, C. L. (1966). An Analysis of Variation Between Upland and Lowland Switchgrass, Panicum Virgatum L., in Central Oklahoma.Ecology, 47 (6), 980-992. doi:10.2307/1935646

Price, D. L. (2014). Predictive Relationships between Plant Morphological Traits and Biomass Yield in Switchgrass. Crop Science, v. 54 (no. 2), pp. 637-630-2014 v.2054 no.2012. doi:10.2135/cropsci2013.04.0272

Robertson, G. P., Hamilton, S. K., Barham, B. L., Dale, B. E., Izaurralde, R. C., Jackson, R. D., . . . Tiedje, J. M. (2017). Cellulosic biofuel contributions to a sustainable energy future: Choices and outcomes. Science, 356 (6345). doi:10.1126/science.aal2324

Sakamoto, T., Miura, K., Itoh, H., Tatsumi, T., Ueguchi-Tanaka, M., Ishiyama, K., Kobayashi, M., Agrawal, G.K., Takeda, S., Abe, K., Miyao, A., Hirochika, H., Kitano, H., Ashikari, M., Matsuoka, M. (2004). An overview of gibberellin metabolism enzyme genes and their related mutants in rice. Plant Physiol, 134(4), $1642-53$.

Shrestha, R., Gomez-Ariza, J., Brambilla, V., \& Fornara, F. (2014). Molecular control of seasonal flowering in rice, arabidopsis and temperate cereals. Annals of Botany, 114, 1445-1458.

Sultan, S. E. (2000). Phenotypic plasticity for plant development, function and life history. Trends in Plant Science, 5 (12), 537-542. doi:https://doi.org/10.1016/S1360-1385(00)01797-0

Takeda, T., Suwa, Y., Suzuki, M., Kitano, H., Ueguchi-Tanaka, M., Ashikari, M., Matsuoka, M. and Ueguchi, C. (2003). The OsTB1 gene negatively regulates lateral branching in rice. The Plant Journal , 33, 513-520. doi:10.1046/j.1365-313X.2003.01648.x

Tan, J., Jin, M., et al. (2016). OsCOL10 , a CONSTANS-Like gene,functions as a flowering time repressor downstream of Ghd7 in rice. Plant and Cell Physiology, 57(4), 798-812.

Tsuji, H., Taoka, K., \& Shimamoto, K. (2010). Regulation of flowering in rice: two florigen genes, a complex gene network, and natural variation. Current Opinion in Plant Biology, 14, 1-8.

Tu, C., Li, T., \& Liu, X. (2019). Genetic and epigenetic regulatory mechanism of rice panicle development. AIP Conference Proceedings, 2079 (1), 020001. doi:10.1063/1.5092379

Ungerer, M. C., Halldorsdottir, S. S., Modliszewski, J. L., Mackay, T. F., \& Purugganan, M. D. (2002). Quantitative Trait Loci for Inflorescence Development in Arabidopsis thaliana. Genetic, 160: 1133-1151.

Van Esbroeck, G. A. (2003). Variation between Alamo and Cave-in-Rock Switchgrass in Response to Photoperiod Extension. Crop Science, v. 43 (no. 2), pp. 639-630-2003 v.2043 no.2002. doi:10.2135/cropsci2003.6390 
Vogel, K. (2000). Improving warm-season forage grasses using selection, breeding, and biotechnology. In: Moore KJ, Anderson BE (eds) Native warm-season grasses: research trends and issues., 30 (CSSA Spec. Publ., Madison, WI), 83-106.

Vogler, D. W., S. Peretz, \& A. G. Stephenson. 1999. Floral plasticity in an iteroparous plant: the interactive effects of genotype, environment, and ontogeny in Campanula rapunculoides(Campanulaceae). American Journal of Botany . 86: 482-494

Vollbrecht, E., Springer, P., Goh, L. et al. (2005). Architecture of floral branch systems in maize and related grasses. Nature, 436, 1119-1126. https://doi.org/10.1038/nature03892

VSN, International. (2019). Genstat for Windows 19th Edition. VSN International, Hemel Hempstead, UK (Web page: Genstat.co.uk).

Wadgymar, S. M., Lowry, D. B., Gould, B. A., Byron, C. N., Mactavish, R. M., \& Anderson, J. T. (2017). Identifying targets and agents of selection: innovative methods to evaluate the processes that contribute to local adaptation. Methods in Ecology and Evolution, 8 (6), 738-749. doi:10.1111/2041-210X.12777

Wang, Y., \& Li, J. (2005). The Plant Architecture of Rice (Oryza sativa). Plant Molecular Biology, 59 (1), 75-84. doi:10.1007/s11103-004-4038-x

Wang, Y., \& Li, J. (2008). Molecular basis of plant architecture.Annu. Rev. Plant Biol, 59: 253-279.

Williams, G. C. (1957). Pleiotropy, natural selection, and the evolution of senescence. Evolution, 11 , 398-411.

Wu, C., Cui, K., Wang, W., Li, Q., Fahad, S., Hu, Q., . . . Peng, S. (2017). Heat-Induced Cytokinin Transportation and Degradation Are Associated with Reduced Panicle Cytokinin Expression and Fewer Spikelets per Panicle in Rice. Frontiers in plant science, 8 , 371-371. doi:10.3389/fpls.2017.00371

Wu, C., Cui, K., Wang, W., Li, Q., Fahad, S., Hu, Q., . . . Peng, S. (2016). Heat-induced phytohormone changes are associated with disrupted early reproductive development and reduced yield in rice.Scientific Reports, 6 , 34978. doi:10.1038/srep34978

Xue, Z., Liu, L., \& Zhang, C. (2020). Regulation of shoot apical meristem and axillary meristem development in plants. Int, J. Mol. Sci., 21 (8), 2917.

Yano, K., Morinaka, Y., Wang, F., Huang, P., Takehara, S., Hirai, T., . . . Matsuoka, M. (2019). GWAS with principal component analysis identifies a gene comprehensively controlling rice architecture.Proceedings of the National Academy of Sciences , 201904964. doi:10.1073/pnas.1904964116

Yu, H., Qiu, Z., Xu, Q., Wang, Z., Zeng, D., Hu, J., . . . Ren, D. (2017). Fine mapping of LOW TILLER 1, a gene controlling tillering and panicle branching in rice. Plant Growth Regulation, 83 (1), 93-104. doi:10.1007/s10725-017-0286-z

Zhang, D., \& Yuan, Z. (2014). Molecular Control of Grass Inflorescence Development. Annual Review of Plant Biology, 65 (1), 553-578. doi:10.1146/annurev-arplant-050213-040104

Zhao, X., Peng, Y., Zhang, J. et al. (2017). Mapping QTLs and meta-QTLs for two inflorescence architecture traits in multiple maize populations under different watering environments. Mol Breeding, 37, 91

Table 1. The latitude, longitude, site code, soil texture, and source of weather data for the 10 experimental fields in the study.

\begin{tabular}{llllll}
\hline Field Site & Site Code & Latitude & Longitude & Soil Texture & Weather Data Source \\
\hline Brookings, SD & BRKG & 44.307 & -96.67 & Clay loam & https://www.ncdc.noaa.gov/cdo-web/results \\
Hickory Corners, MI & KBSM & 42.42 & -85.37 & Loam & https://tter.kbs.msu.edu/datatables/7 \\
Lincoln, NE & LINC & 41.154 & -96.42 & Loam & https://www.ncdc.noaa.gov/cdo-web/results
\end{tabular}




\begin{tabular}{llllll}
\hline Field Site & Site Code & Latitude & Longitude & Soil Texture & Weather Data Source \\
\hline Manhattan, KS & MNHT & 39.141 & -96.64 & Sandy loam & mesonet.k-state.edu/weather/historical \\
Columbia, MO & CLMB & 38.897 & -92.22 & Loam & http://agebb.missouri.edu/weather/history/i \\
Stillwater, OK & STIL & 35.991 & -97.05 & Sandy loam & https://www.mesonet.org/index.php/weath \\
Overton, TX & OVTN & 32.303 & -94.98 & Sandy loam & https://www.ncdc.noaa.gov/cdo-web/results \\
Temple, TX & TMPL & 31.043 & -97.35 & Clay & https://www.ars.usda.gov/plains-area/templ \\
Austin, TX & PKLE & 30.384 & -97.73 & Clay & https://www.ncdc.noaa.gov/cdo-web/results \\
Kingsville, TX & KING & 27.55 & -97.88 & Sandy clay loam & https://www.ncdc.noaa.gov/cdo-web/results \\
\hline
\end{tabular}

Table 2. Narrow-sense heritability $\left(h^{2}\right)$, and its one standard error $( \pm 1 \mathrm{SE})$, for panicle length $(\mathrm{PL})$, primary branching number $(\mathrm{PBN})$, and secondary branching number $(\mathrm{SBN})$ at each of the 10 field sites (ordered from north to south).

\begin{tabular}{llll}
\hline Sites/Traits & PL & PBN & SBN \\
\hline BRKG & $0.55 \pm 0.07$ & $0.64 \pm 0.06$ & $0.40 \pm 0.08$ \\
KBSM & $0.71 \pm 0.06$ & $0.62 \pm 0.07$ & $0.56 \pm 0.07$ \\
LINC & $0.58 \pm 0.07$ & $0.65 \pm 0.07$ & $0.47 \pm 0.08$ \\
MNHT & $0.38 \pm 0.08$ & $0.66 \pm 0.06$ & $0.38 \pm 0.08$ \\
CLMB & $0.57 \pm 0.07$ & $0.64 \pm 0.07$ & $0.15 \pm 0.08$ \\
STIL & $0.20 \pm 0.08$ & $0.13 \pm 0.08$ & $0.02 \pm 0.07$ \\
OVTN & $0.44 \pm 0.08$ & $0.63 \pm 0.06$ & $0.62 \pm 0.07$ \\
TMPL & $0.49 \pm 0.08$ & $0.54 \pm 0.07$ & $0.30 \pm 0.08$ \\
PKLE & $0.24 \pm 0.09$ & $0.45 \pm 0.09$ & $0.29 \pm 0.09$ \\
KING & $0.47 \pm 0.08$ & $0.58 \pm 0.07$ & $0.48 \pm 0.08$ \\
\hline
\end{tabular}

Table 3. The phenotypic correlation and genetic correlation between panicle traits among sites and across sites. PL, panicle length, PBN, primary branching number, and SBN, secondary branching number.

\begin{tabular}{|c|c|c|c|c|c|c|}
\hline Sites & & Phenotypic correlation & Phenotypic correlation & Phenotypic correlation & Genetic correlation & $\mathrm{Ge}$ \\
\hline & & $\mathrm{PL}$ & $\mathrm{PBN}$ & SBN & PL & $\mathrm{PB}$ \\
\hline & PL & 1 & - & - & 1 & - \\
\hline \multirow[t]{3}{*}{ BRKG } & $\mathrm{PBN}$ & 0.41 & 1 & - & 0.68 & 1 \\
\hline & SBN & 0.42 & 0.39 & 1 & 0.82 & 0.7 \\
\hline & PL & 1 & - & - & 1 & - \\
\hline \multirow[t]{3}{*}{ KBSM } & $\mathrm{PBN}$ & 0.30 & 1 & - & 0.44 & 1 \\
\hline & SBN & 0.42 & 0.54 & 1 & 0.62 & 0.7 \\
\hline & $\mathrm{PL}$ & 1 & - & - & 1 & - \\
\hline \multirow[t]{3}{*}{ LINC } & $\mathrm{PBN}$ & 0.43 & 1 & - & 0.49 & 1 \\
\hline & SBN & 0.52 & 0.60 & 1 & 0.79 & 0.8 \\
\hline & PL & 1 & - & - & 1 & - \\
\hline \multirow[t]{3}{*}{ MNHT } & $\mathrm{PBN}$ & 0.56 & 1 & - & 0.62 & 1 \\
\hline & SBN & 0.68 & 0.67 & 1 & 0.74 & 0.8 \\
\hline & PL & 1 & - & - & 1 & - \\
\hline \multirow[t]{3}{*}{ CLMB } & $\mathrm{PBN}$ & 0.36 & 1 & - & 0.50 & 1 \\
\hline & SBN & 0.40 & 0.50 & 1 & 0.88 & 0.9 \\
\hline & PL & 1 & - & - & 1 & - \\
\hline \multirow[t]{2}{*}{ STIL } & PBN & 0.42 & 1 & - & 0.35 & 1 \\
\hline & SBN & 0.42 & 0.57 & 1 & - & - \\
\hline
\end{tabular}




\begin{tabular}{|c|c|c|c|c|c|c|}
\hline Sites & & Phenotypic correlation & Phenotypic correlation & Phenotypic correlation & Genetic correlation & $\mathrm{Gel}$ \\
\hline & $\mathrm{PL}$ & 1 & - & - & 1 & - \\
\hline \multirow[t]{3}{*}{ OVTN } & PBN & 0.52 & 1 & - & 0.58 & 1 \\
\hline & SBN & 0.59 & 0.58 & 1 & 0.72 & 0.7 \\
\hline & PL & 1 & - & - & 1 & - \\
\hline \multirow[t]{3}{*}{ TMPL } & PBN & 0.35 & 1 & - & 0.54 & 1 \\
\hline & SBN & 0.53 & 0.46 & 1 & 0.69 & 0 \\
\hline & PL & 1 & - & - & 1 & - \\
\hline \multirow[t]{3}{*}{ PKLE } & PBN & 0.27 & 1 & - & 0.61 & 1 \\
\hline & SBN & 0.21 & 0.51 & 1 & 0.47 & 0.7 \\
\hline & PL & 1 & - & - & 1 & - \\
\hline \multirow[t]{2}{*}{ KING } & PBN & 0.52 & 1 & - & 0.56 & 1 \\
\hline & SBN & 0.63 & 0.61 & 1 & 0.70 & 0.7 \\
\hline \multirow[t]{3}{*}{ Across Sites } & $\mathrm{PL}$ & 1 & - & - & 1 & - \\
\hline & PBN & 0.50 & 1 & - & 0.03 & 1 \\
\hline & SBN & 0.55 & 0.61 & 1 & 0.38 & 0.7 \\
\hline
\end{tabular}

Table 4. The identified QTL, along with their marker name (chromosome with physical distance in mega base pair), maximum LOD values, and flanking markers with a LOD drop of 1.5 for panicle morphology traits (PL: panicle length; PBN: number of primary branches; SBN: number of secondary branches). The presence of genotype by environmental interaction is marked as 'Yes' or 'No' in column Q x E. The overlapping QTL confidence interval between traits indicates pleiotropic effect, and what other traits (FL50, TC, and BIO) have pleiotropy with panicle traits at each identified QTL position is marked in column Pleiotropy. FL50, $\mathrm{TC}$, and $\mathrm{BIO}$ are flowering time, tiller count and biomass at the end of season, respectively.

\begin{tabular}{|c|c|c|c|c|c|c|c|}
\hline trait & QTL & MARKER & LOD & Left flanking marker & Right flanking_marker & $\mathrm{QxE}$ & Pleiotropy \\
\hline$\overline{\mathrm{PL}}$ & $2 \mathrm{~K} @ 77.89$ & Chr02K_62.598826 & 6.18 & Chr02K_60.739957 & Chr02K_64.045891 & No & $\mathrm{BIO}$ \\
\hline PL & $3 \mathrm{~N} @ 62.06$ & Chr03N_26.099983 & 4.29 & Chr03N_24.521536 & Chr03N_30.30364 & Yes & $\mathrm{BIO}$ \\
\hline PL & $4 \mathrm{~K} @ 26.26$ & Chr04K_13.041487 & 4.66 & Chr04K_9.916183 & Chr04K_29.613196 & No & FL50 \\
\hline $\mathrm{PL}$ & $5 \mathrm{~K} @ 76.02$ & Chr05K_56.620419 & 4.82 & Chr05K_44.678143 & Chr05K_58.488157 & No & \\
\hline $\mathrm{PL}$ & $5 \mathrm{~N} @ 36.27$ & Chr05N_16.511689 & 3.63 & Chr05N_11.735767 & Chr05N_47.154718 & No & \\
\hline $\mathrm{PL}$ & $6 \mathrm{~N} @ 54.19$ & Chr06N_48.768076 & 3.56 & Chr06N_43.871788 & Chr06N_51.935176 & Yes & \\
\hline PL & $9 \mathrm{~N} @ 38.02$ & Chr09N_18.617122 & 5.82 & Chr09N_10.880731 & Chr09N_20.831824 & No & FL50, TC, BIO \\
\hline $\mathrm{PBN}$ & $2 \mathrm{~K} @ 74.02$ & Chr02K_59.503978 & 4.09 & Chr02K_56.436103 & Chr02K_63.664705 & No & $\mathrm{BIO}$ \\
\hline $\mathrm{PBN}$ & $2 \mathrm{~N} @ 66.12$ & Chr02N_55.500715 & 5.52 & Chr02N_50.387752 & Chr02N_56.445418 & No & FL50 \\
\hline PBN & $3 \mathrm{~K} @ 38$ & Chr03K_17.77051 & 8.83 & Chr03K_13.323286 & Chr03K_20.786505 & Yes & FL50, TC \\
\hline PBN & $5 \mathrm{~K} @ 14.06$ & Chr05K_7.188103 & 4.90 & Chr05K_4.388419 & Chr05K_8.204815 & Yes & \\
\hline PBN & $5 \mathrm{~N} @ 84.04$ & Chr05N_64.047349 & 4.73 & Chr05N_60.974614 & Chr05N_65.990782 & No & FL50, TC \\
\hline PBN & $7 \mathrm{~N} @ 54.06$ & Chr07N_49.904749 & 4.17 & Chr07N_49.035214 & Chr07N_49.904749 & Yes & FL50 \\
\hline PBN & $9 \mathrm{~N} @ 26.03$ & Chr09N_12.531268 & 4.89 & Chr09N_7.913256 & Chr09N_21.588445 & No & FL50 \\
\hline SBN & $2 \mathrm{~N} @ 72.03$ & Chr02N_58.696003 & 9.46 & Chr02N_54.556579 & Chr02N_60.798034 & No & \\
\hline SBN & $5 \mathrm{~K} @ 95.5$ & Chr05K_60.232411 & 6.22 & Chr05K_58.583292 & Chr05K_60.232411 & No & \\
\hline SBN & 9K@51.96 & Chr09K_24.465322 & 10.37 & Chr09K_19.959778 & Chr09K_28.697896 & Yes & \\
\hline SBN & $9 \mathrm{~N} @ 38.02$ & Chr09N_18.617122 & 9.29 & Chr09N_17.684245 & Chr09N_19.333648 & No & $\mathrm{TC}, \mathrm{BIO}$ \\
\hline
\end{tabular}

Table 5. The environmental factor(s) selected by best subset general linear regression model based on Bayesian information criteria for the effects of QTL by environment interaction (QTL x E) for panicle traits. PL: panicle length; PBN: number of primary branches; SBN: number of secondary branches. A x B and C $\mathrm{x}$ D denote the cross from AP13 x DAC and WBC x VS16, respectively. Tmean, Rainfall, DL, and SRAD 
are annual mean temperature, total rainfall, day length (photoperiod) and average solar radiation in 2016, respectively. * indicates the environmental factor that was considered as the candidate factor for the QTL $\mathrm{x}$ E based on the model.

\begin{tabular}{lllllll}
\hline Trait & QTL & Cross & Tmean & Rainfall & DL & SRAD \\
\hline PL & $3 \mathrm{~N} @ 62.06$ & A $\times$ B & & & & \\
& & C $\times$ D & $*$ & & $*$ & \\
PL & $6 \mathrm{~N} @ 54.19$ & A $\times$ B & & $*$ & $*$ & \\
& & C $\times$ D & $*$ & $*$ & & \\
PBN & $3 \mathrm{~K} @ 38$ & A $\times$ B & $*$ & & $*$ & \\
& & C $\times$ D & & $*$ & $*$ & \\
PBN & $5 \mathrm{~K} @ 14.06$ & A $\times$ B & & $*$ & $*$ & \\
& & C $\times$ D & $*$ & $*$ & $*$ \\
PBN & $7 \mathrm{~N} @ 54.06$ & A $\times$ B & $*$ & $*$ & $*$ & \\
& & C $\times$ D & & & & $*$ \\
SBN & $9 \mathrm{~K} @ 51.96$ & A $\times$ B & $*$ & & & \\
& & C $\times$ D & $*$ & & & \\
\hline
\end{tabular}

\section{Hosted file}

Figures_PCE.pdf available at https://authorea.com/users/373444/articles/491132-qtl-xenvironment-interactions-for-panicle-traits-in-switchgrass-panicum-virgatum 